\title{
Tumor cell heterogeneity and resistance; report from the 2018 Coffey-Holden Prostate Cancer Academy Meeting
}

\author{
Andrea K. Miyahira ${ }^{1}$ (D) | Robert B. Den ${ }^{2}$ | Maria I. Carlo ${ }^{3}$ | Renée de Leeuw \\ Thomas A. Hope ${ }^{5,6,7}$ | Fatima Karzai ${ }^{8}$ | Rana R. McKay9 | Simpa S. Salami ${ }^{10,11}$ | \\ Jonathan W. Simons ${ }^{1}$ | Kenneth J. Pienta ${ }^{12,13,14}$ | Howard R. Soule ${ }^{1}$ \\ ${ }^{1}$ Prostate Cancer Foundation, Santa Monica, California \\ ${ }^{2}$ Department of Radiation Oncology, Thomas Jefferson University, Philadelphia, Pennsylvania \\ ${ }^{3}$ Department of Medicine, Memorial Sloan Kettering Cancer Center, New York, New York \\ ${ }^{4}$ Department of Pathology, College of Medicine, University of Illinois at Chicago, Chicago, Illinois \\ ${ }_{5}^{5}$ Department of Radiology and Biomedical Imaging, University of California San Francisco, San Francisco, California \\ ${ }^{6}$ Department of Radiology, San Francisco VA Medical Center, San Francisco, California \\ 7 UCSF Helen Diller Family Comprehensive Cancer Center, University of California San Francisco, San Francisco, California \\ ${ }^{8}$ Genitourinary Malignancies Branch, Center for Cancer Research, National Cancer Institute, National Institutes of Health, Bethesda, Maryland \\ ${ }^{9}$ Department of Medicine, Division of Hematology/Oncology, University of California San Diego, San Diego, California \\ ${ }^{10}$ Department of Urology, University of Michigan Health System, Ann Arbor, Michigan \\ 11 University of Michigan Rogel Cancer Center, Ann Arbor, Michigan \\ ${ }_{12}$ Department of Oncology, Sidney Kimmel Comprehensive Cancer Center, The Johns Hopkins School of Medicine, Baltimore, Maryland \\ ${ }^{13}$ Department of Urology, The James Buchanan Brady Urological Institute, Baltimore, Maryland \\ ${ }^{14}$ Department of Pharmacology and Molecular Sciences, The Johns Hopkins School of Medicine, Baltimore, Maryland
}

\section{Correspondence}

Andrea K. Miyahira, Prostate Cancer

Foundation, 1250 4th Street, Santa Monica, CA 90401.

Email: amiyahira@pcf.org
Introduction: The 2018 Coffey-Holden Prostate Cancer Academy (CHPCA) Meeting, "Tumor Cell Heterogeneity and Resistance," was held in Los Angeles, California from June 21 to 24, 2018.

Methods: The CHPCA Meeting is a unique, discussion-oriented scientific conference convened annually by the Prostate Cancer Foundation (PCF), which focuses on the most critical topics in need of further study to advance the treatment of lethal prostate cancer. The 6th Annual CHPCA Meeting was attended by 70 investigators and concentrated on prostate cancer heterogeneity and treatment resistance.

Results: The meeting focused on topics including: recognition of tumor heterogeneity, molecular drivers of heterogeneity, the role of the tumor microenvironment, the role of heterogeneity in disease progression, metastasis and treatment resistance, clinical trials designed to target resistance and tumor heterogeneity, and immunotherapeutic approaches to target and overcome tumor heterogeneity.

Discussion: This review article summarizes the presentations and discussions from the 2018 CHPCA Meeting in order to share this knowledge with the scientific community and encourage new studies that will lead to improved treatments and outcomes for men with prostate cancer.

KEYWORDS

biomarkers, cancer immunotherapy, molecular imaging, therapeutics, tumor genomics 


\section{1 | INTRODUCTION}

The Prostate Cancer Foundation (PCF) is the largest non-profit foundation in the world that funds research focused on improving the understanding of prostate cancer biology and advancing new life-extending and life-improving treatments for patients stricken with the most aggressive forms of this disease. As a critical supplement to this effort, PCF convenes several scientific conferences every year and has a large program dedicated to facilitating global knowledge exchange and the development of new collaborations and research initiatives.

For six consecutive years, PCF has convened the Coffey-Holden Prostate Cancer Academy (CHPCA) Meeting, an annual "think tank" conference, which gathers $\sim 75$ investigators to discuss the most critical topics necessary for making a near-term impact on the understanding and treatment of lethal prostate cancer. ${ }^{1-5}$ This meeting is uniquely designed to promote deep and ample discussion and brainstorming of necessary next steps by structuring sessions into short talks with lengthy discussion times. This meeting also supports a major goal of PCF to promote career development of young investigators by mandating that the organizing committee and approximately half of attendees be early career investigators. The CHPCA Meeting follows the discussion-oriented structure of the former $\mathrm{NCl}$ Prouts Neck Meetings on Prostate Cancer, ${ }^{6}$ and is named in honor of the prostate cancer research pioneers, Dr Stuart Holden and the late Dr Donald Coffey.

The 2018 CHPCA Meeting was held from June 21-24, 2018, in Los Angeles, California, and was themed "Tumor Cell Heterogeneity and Resistance." 70 investigators attended, including 45 young investigators. Talks and discussions centered on critical topics surrounding the biology of prostate cancer heterogeneity and the impact of heterogeneity on treatment outcomes, including recognition of tumor heterogeneity, molecular drivers of heterogeneity, the role of the tumor microenvironment, the role of heterogeneity in disease progression, metastasis and treatment resistance, clinical trials designed to target resistance and tumor heterogeneity, and immunotherapeutic approaches to target and overcome tumor heterogeneity.

\section{2 | UNDERSTANDING PROSTATE CANCER HETEROGENEITY}

Prostate cancer exists in a clinical continuum, ranging from low to high grade disease, localized to metastatic disease, and hormone-sensitive to castration-resistant disease. The evolution of the disease from one end of the spectrum to another is associated with continuing changes in the genome and emergence of new clones and subclones under the influence of external pressures. There are unique molecular characteristics involved in cancer cellular processes such as invasion, migration, and metastasis that can be measured to classify prostate cancer into subtypes and these may define cancer cell vulnerability or resistance to treatment pressure. Importantly, therapeutic pressures cause cancers to lose and/or gain molecular alterations as new subtypes emerge due to new mutations or epigenetic changes. ${ }^{7,8}$ Additionally, pre-existing genetic variants with resistance characteristics may only become evident or dominant due to treatment selection pressure. Tumor adaptation due to pre-existing genetic variation is likely faster than adaptation through de novo mutations or epigenetic alterations, as beneficial mutations are immediately available in the new environment (ie, treatment), and may also start at higher frequencies.

Numerous questions exist surrounding the biology of tumor heterogeneity, including a better understanding of which biologic features of heterogeneity matter for treatment and outcomes, what are the critical molecular characteristics of heterogeneity, what are the roles of truncal versus subclonal genomic alterations, and how to identify relevant rare clones that may later become a dominant, treatment-resistant form of disease. The non-treatment related drivers of heterogeneity are also unclear, including the roles for genomic instability, regional hypoxia, field effects, immune, stromal and other heterogeneous factors within the tumor microenvironment, and immune pressure. Understanding how to successfully treat heterogeneous disease within a patient is ultimately necessary for developing cures for advanced prostate cancer.

\section{3 | HETEROGENEITY OF MULTI-FOCAL PRIMARY PROSTATE CANCER}

One of the hallmarks of prostate cancer is that most men harbor multiple areas or foci of primary disease, which led to the routine use of sextant biopsies to ensure adequate sampling of the gland. ${ }^{9}$ It is critical to accurately identify tumor foci with dominant or aggressive potential in any given prostate as these sites contribute to the subclonal complexity of metastatic disease. ${ }^{10,11}$

Heterogeneity between different tumor foci within the prostate has been demonstrated. ${ }^{12,13}$ These issues of prostate cancer multifocality and multiclonality confound interpretations of tissuebased genomic and transcriptomic biomarkers and commercially available prognostic tests, which impact clinical decision making. ${ }^{13,14}$ While tissue-based markers assessed on low grade disease may predict the concomitant presence of an undersampled high grade disease, emerging data from Wei et $\mathrm{al}^{13}$ and Salami et al (unpublished) suggest that they may not predict the presence of an unsampled high grade disease. Liquid biopsy approaches such as those based on urine RNA transcripts (eg, TMPRSS2:ERG, PCA3, SChLAP1) may help circumvent the problems imposed by tumor multifocality and heterogeneity. Lessons from breast cancer suggest that no single modality is perfect, but rather that multiple complementary approaches may be needed. ${ }^{15}$ In prostate cancer, the combination of imaging (MRI, PET), liquid biopsy (urine- and blood-based), and tissue biopsy approaches may provide the best opportunity to improve early detection of aggressive prostate cancer by overcoming tumor multifocality and multiclonality issues. While validation in larger cohorts with long term patient outcomes is critical, these findings highlight the importance and need for more comprehensive approaches. 


\section{4 | GENOMIC DRIVERS OF PROSTATE CANCER DIVERSITY AND TREATMENT RESISTANCE}

Prostate cancer is driven by the activity of the androgen receptor (AR), a transcription factor of the steroid nuclear receptor family. ${ }^{16}$ This seminal finding was originally noted by Charles Huggins in the early $1940 s^{17}$ and 80 years later, whole exome and whole genome sequencing efforts continue to provide new insights into AR biology and its regulation in prostate cancer.

Alterations that maintain activity of the AR pathway have been found to drive the vast majority of castration-resistant prostate cancer (CRPC) cases. Whole-exome and transcriptome sequencing have found that AR pathway alterations are apparent in $~ 71 \%$ of metastatic CRPC (mCRPC) cases, primarily AR gene amplifications and mutations. ${ }^{18}$ Recent whole genome sequencing studies have identified an expanded role for AR alterations and continued AR activity in driving CRPC, as tandem duplications of enhancer elements located upstream of $A R$ that act as critical drivers of AR expression and AR-targeted therapy resistance were found in $70-87 \%$ of CRPC cases, compared with $<2 \%$ of primary prostate cancer cases. ${ }^{19-21}$ These sites are likely regulated by epigenetic alterations, as the genomic sequencing peak coincides with H3K27ac signal. Tandem duplications of enhancers of MYC and FOXA1 were also found to be common, ${ }^{19,21}$ highlighting the importance of evolutionally driven enhancers in CRPC. The formation of tandem enhancers appears to be regulated by proteins critical to DNA repair, including CDK12. ${ }^{22}$ Recent studies suggest that bi-allelic loss of CDK12 may confer sensitivity to checkpoint immunotherapy, due to increased levels of fusion neoantigens generated by the tandem duplicator phenotype. ${ }^{22}$ Clinical trials to directly test this hypothesis are underway.

Studies have suggested that prostate cancer heterogeneity emerges early in tumorigenesis and is further selected for by treatment. A neoadjuvant trial of ADT+abiraterone acetate in localized high-risk prostate cancer found that while some alterations develop in response to treatment, some of the same alterations, such as RB1 loss, may have been present in untreated tumors and selected for by the treatment. ${ }^{23}$ Studies comparing matched pre- and post-treatment cancer tissue are necessary to determine the frequency of this occurrence. An ongoing study at the National Cancer Institute where pre-treatment biopsy is obtained using magnetic resonance imaging/ ultrasound (MRI/US) fusion guidance and compared with posttreatment radical prostatectomy tissue will be informative (NCT02430480). Preliminary results from this study have identified some differential characteristics between responders and nonresponders to ADT + abiraterone acetate independent of Gleason score. Non-responders were more likely to have intraductal carcinoma, have more heterogeneous somatic copy number alterations, and be ERG-positive. Not surprisingly, intra-person phenotypic heterogeneity in response was observed in some patients. Imaging features that can differentiate responders from non-responders in this trial are under study. The molecular correlates from this study will be insightful.
Further studies are needed to determine what drives the development of tumor heterogeneity and subclonal evolution during earlier stages of tumor progression.

There is abundant effort to characterize each individual patient's cancer genome using DNA and RNA based approaches to identify drivers of cancer and inform therapy selection. ${ }^{24}$ Despite initial optimism, sequencing efforts have led to the discovery of laundry lists of mutations and alterations which have no functional consequence, and of "druggable" targets which fail to yield clinically meaningful results with the administration of targeted therapies. This suggests that further proteomic ${ }^{25}$ and epigenomic ${ }^{26}$ information is critical to yield insight into cancer dependencies and improve the selection of effective treatments. Additionally, rigorous protocols and optimized methods to define informative markers are needed to facilitate precision medicine treatment efforts in the larger cancer community.

\section{5 | HETEROGENEITY OF AR-INDEPENDENT CRPC}

Tumor heterogeneity is a large contributor to response or resistance to AR-targeted therapy. Possible outcomes following AR-targeted therapy include a durable complete response, or varied levels and durations of responses followed by resistance through AR-driven or AR-independent pathways. ${ }^{27}$ AR-independent/indifferent CRPC subtypes include neuroendocrine prostate cancer (NEPC) (some of which still express $A R$ ), and subtypes negative for both $A R$ and neuroendocrine (NE) markers ("double-negative"). ${ }^{28,29}$ A study by investigators at the University of Washington and Fred Hutchinson Cancer Research Center used tumors acquired from rapid autopsies and found that from 1998 to 2011, over $88 \%$ of lethal prostate cancer cases were AR-positive, 5.4\% were AR-negative/NE-positive, and 6.3\% were double-negative. However, from 2012 to 2016, following the additions of the more potent AR-axis inhibitors abiraterone acetate and enzalutamide to the prostate cancer treatment arsenal, the prevalence of AR-negative prostate cancer among lethal cases dramatically increased, to $13.3 \%$ being ARnegative/NE-positive and $23.3 \%$ double-negative. ${ }^{28}$ These findings suggest that evolution under treatment pressure is a large contributor to the development of AR-null prostate cancer phenotypes. A study by the PCF West Coast Dream team recently reported that $17 \%$ of progressive, $\mathrm{mCRPC}$ had the phenotype of treatment-emergent small-cell neuroendocrine prostate cancer ( $t$ $\mathrm{SCNC}) .{ }^{30}$ Additional studies are necessary to confirm the hypothesis that the landscape of CRPC phenotypes has changed in response to more potent AR pathway inhibition, and to better define these subtypes molecularly and clinically.

Understanding the mechanisms that drive AR-independent disease progression is critical toward developing more effective therapies. AR-positive/NE-positive, AR-positive/NE-negative, ARnegative/NE-positive, and double-negative mCRPC can be 
differentiated by gene expression patterns. ${ }^{28}$ Double-negative mCPRC were found to exhibit elevated FGF and MAPK pathway activity. ${ }^{28}$ FGF/MAPK pathway blockade had on-target and antitumor effects in double-negative mCRPC patient-derived xenograft (PDX) models, and FGFR-inhibition was found to synergize with enzalutamide, supporting the FGF pathway as a driver of AR-therapy resistance. Other known mechanisms of resistance to complete ARpathway blockade include loss of p53, Rb1, and/or PTEN. ${ }^{7}$ Whether double-negative $\mathrm{mCPRC}$ may be an intermediate/dedifferentiated point and could transition into NEPC deserves further study. Preliminary data presented at this meeting indicated that a phenotype resembling squamous cell carcinoma may also develop in response to AR blockade. The mechanism of development of this subtype and potential therapeutic approaches are currently unknown. Altogether, there is likely a continuum of AR-indifferent and AR-low/null mCRPC subtypes. The number of possible lineage pathways remains to be determined.

\section{6 | EPIGENETIC ALTERATIONS IN CRPC}

The role of epigenetic heterogeneity in tumor biology and treatment responses is of critical interest. Studies into epigenetic hot spots demonstrate that many hypermethylation changes are conserved between different metastases and are recurrently present across multiple patients, ${ }^{31}$ suggesting that these sites have the potential for producing selectable driver events. One such example is PRAC1, a gene exclusively expressed in prostate, colon, and rectal tissue (Haffner et al, unpublished, ${ }^{32}$ ). PRAC1 can be epigentically silenced in CRPC and in vitro and in vivo studies demonstrate that loss of PRAC1 leads to castrate resistant growth (Haffner et al, unpublished). Whether such hypermethylation changes may be reversed by treatment or serve as biomarkers is of question. The relationship between hypermethylation changes and the genomic background of the tumor also deserves further study.

While hypermethylation suggests potentially druggable targets consequences of hypomethylation may include altering of the immune milieu. Global loss of epigenetic repressive markers have led to increased activity of retrotransposons such as LINE1 and ALU1, ${ }^{33}$ genomic instability, ${ }^{34}$ and expression of neoantigens including NY-ESO-1 and MAGE. $^{35}$ This has the potential to be therapeutically exploited, as pharmacologically induced hypomethylation can alter the immune microenvironment, increase interferon signaling, and improve lymphocytic infiltration into tumors. ${ }^{36,37}$ These effects suggest synergy may be achieved with hypomethylation-inducing agents and checkpoint inhibitors, and warrant further study. A constitutive "extreme hypomethylation" phenotype with associated profound changes in the intratumoral immune microenvironment has been recently observed in testicular germ cell tumors. ${ }^{38}$ In such hypomethylated tumors, endogenous retrovirus expression is greatly increased resulting in enhanced interferon type I responses. This demonstrates a previously unrecognized link between cancer cell specific epigenetic alterations and the tumor immune microenvironment.

\section{7 | SYNTHETIC ESSENTIALITY: TARGETING GENETIC HETEROGENEITY IN PROSTATE CANCER}

Many therapeutic strategies are being explored to target cancer genetic alterations. In oncogene addiction, cancer cells are physiologically dependent on the continued activity of oncogenes to maintain a malignant phenotype. ${ }^{39}$ Clinical success has been achieved in targeting oncogenes as exemplified by targeting AR amplification with enzalutamide. However, there are still many undruggable oncogenes or tumor suppressor genes. Another concept is synthetic lethality, in which tumor cells that have lost activity of one molecular pathway become highly dependent on a second related pathway, which can be therapeutically targeted. ${ }^{40}$ For example, clinical success has been seen with PARP inhibitors in patients with tumors with homologous recombination deficiency (HRD). ${ }^{41}$ A similar concept is "collateral lethality," in which passenger genomic events in tumor cells can create unintended vulnerabilities, particularly in the deletion of redundant essential housekeeping genes. ${ }^{42,43} \mathrm{~A}$ related concept is "synthetic essentiality," in which genes that may be deleted in some cancers, are almost always retained in the context of a specific tumor suppressor deficiency, and may represent therapeutic vulnerabilities. ${ }^{44}$ Synthetic essential genes may be identified with the aid of available cancer genome and clinical databases, by examining for genes that exhibit mutually exclusive deletion patterns. ${ }^{44}$ Zhao et al identified the epigenetic regulator CHD1 as being deleted in some prostate cancers in a mutually exclusive manner with PTEN. ${ }^{45} \mathrm{CHD} 1$ was further demonstrated to be a possible therapeutic target in PTEN-deficient prostate cancer models. ${ }^{45}$ In PTEN-deficient breast and prostate cancer, CHD1 is a synthetic-essential gene, which when degraded via the PTEN-AKT-GSK3 $\beta$ axis, suppressed cell proliferation, and cell survival. ${ }^{45}$ Studies to validate whether CHD1 is an active therapeutic target in PTEN-deficient human cancer may be warranted. Using the concept of synthetic essentiality, tumor suppressor deficiencies can be targeted for clinical benefit.

\section{8 | SINGLE CELL PROTEOGENOMICS}

Because of the high levels of tumor cell genomic, molecular, and phenotypic heterogeneity, single cell analyses can greatly enrich understanding of tumor cell biology and function. Kuhn and colleagues have developed a high definition single cell analysis workflow to analyze morphology, gene expression, and genomics of single circulating tumor cells (CTCS). This technology has been applied to over 15000 samples from over 4000 patients with prostate and other cancers. CTCs were found to have heterogeneous morphology, belying the importance of single cell analyses to understand biologies that correspond with one another and biologies that are exclusive or incompatible. ${ }^{46}$ A slide-based approach using time of flight mass cytometry (CyTOF), which enables subcellular resolution of up to 35 proteomic parameters simultaneously, is now being applied to add proteomics information to single cell CTC analyses. 
CTCs have been demonstrated to have utility as predictive biomarkers for treatment responses. The absence or presence of nuclear AR-V7 in CTCs has been found to be predictive for better survival if treated with AR-targeted therapy versus taxane chemotherapy, respectively, as second line treatment for progressive mCRPC. ${ }^{47}$ This test has been commercialized and is now being used in the clinic.

The Blood Profiling Atlas in Cancer (BloodPAC) Consortium is a Cancer Moonshot inspired program that aims to accelerate the development and validation of liquid biopsy assays for various types of cancer. Three pilot projects are underway to improve and validate high-throughput liquid biopsy methodologies, including a PCF-funded project that will determine whether variations in blood collection protocols, such as sampling intervals, time of day, draw order, fasting status, and anti-emetics, affect prostate cancer CTC analytical results.

\section{9 | IMAGING APPROACHES FOR IDENTIFYING AGGRESSIVE PROSTATE CANCER AND TUMOR HETEROGENEITY}

Conventional imaging using computed tomography (CT) has limited ability to characterize heterogeneity beyond the location of metastatic lesions. There is great interest in leveraging more advanced imaging techniques that enable better lesion characterization. Newer imaging approaches including PSMA PET and multi-parametric MRI have significantly changed our paradigm for detecting and understanding prostate cancer heterogeneity. Though primarily designed for evaluating the location and extent of disease, imaging has the potential to provide biologically relevant information such as tumor aggressiveness.

PSMA (prostate specific membrane antigen) is highly and specifically expressed on the surface of prostate cancer cells, and is positively correlated with disease progression. PSMA PET has led to improvement in estimating the burden of disease in primary and recurrent prostate cancer with high sensitivity and specificity. ${ }^{48,49}$ Although early data suggests that PSMA PET may be useful for predicting disease response, ${ }^{50}$ it is unclear how to interpret the intensity of PSMA expression in relation to Gleason score, tumor biology, tumor microenvironment, and androgen dependency. In a case series of men with metastatic prostate cancer treated with frontline and salvage chemotherapy, concordance between PSA and PSMA PET response was reported. ${ }^{51}$ Although PSMA is expressed on $>90 \%$ of prostate cancer cases, expression is linked to AR-signaling and can be heterogeneous within an individual. PSMA PET may not be useful in all patients, including those with NEPC or ductal carcinoma, as PSMA expression can be lost on these prostate cancer subtypes. A study comparing PSMA-targeted ${ }^{18} \mathrm{~F}$-DCFBC PET/CT and ${ }^{18} \mathrm{~F}$-sodium fluoride (NaF) PET/CT for detection of bone lesions in patients with metastatic prostate cancer, found that while detection of putative lesions between the two technologies were largely concordant, the majority of lesions were detected by one modality but not the other. ${ }^{52}$ Because NaF PET is an indicator of osteoblastic activity and not a direct measure of tumor burden, it will be important to understand how treated lesions versus viable lesions are differentially identified by these modalities. Discordance has also been observed between PSMAPET and ${ }^{18}$ F-FDG PET imaging, further suggesting biological heterogeneity across different lesions. ${ }^{53}$ The underlying biology and clinical implications of this discordance are yet unclear. Prospective studies are needed to inform how best to change patient management based on PSMA PET and other imaging findings. PCF has previously published a report from a working group meeting held on issues surrounding PSMA as an imaging and therapeutic target; issues surrounding the heterogeneity of PSMA expression were a major topic of discussion. ${ }^{54}$

Multi-parametric MRI measures a number of different functional and anatomical features of tissues such as vascularization and diffusion of water molecules, providing staging and possibly cancer biology information. In a recent randomized trial of targeted biopsy versus standard biopsy, the MRI PI-RADS score was found to be correlated with clinically significant prostate cancer defined as Gleason $\geq 7(12 \%$ for PI-RADS 3, 60\% for PI-RADS 4, and $83 \%$ for PI-RADS 5). ${ }^{55}$ However, the accuracy of MRI as a predictor of long term oncological outcomes is unknown. In an unpublished work $(n=612)$ by Faena and colleagues at the University of California Los Angeles (UCLA), PI-RADS on MRI was shown to predict biochemical recurrence, a surrogate for disease aggressiveness. Additionally, Salmasi and colleagues have demonstrated that Oncotype Dx GPS correlated positively with PIRADS, suggesting that MRI may be providing some biological information. ${ }^{56}$ However, the biological basis of cancer visibility on MRI is poorly understood. In work examining histological appearance of the tumor, only $17 \%$ of lesions with cribriform pattern 4 were visible on $\mathrm{MRI}^{57} \mathrm{~A}$ study that compared 49 radiomic features (diffusion weighted (DWI), Ktrans etc.) to RNA expression data from $17 \mathrm{MRI}$ targeted biopsies from six patients found that distinct radiomic features in the transition and peripheral zones correlated with gene signatures, suggesting the possibility of a field effect. ${ }^{58}$

One issue with DWI MRI is the technical difficulty in implementing a robust and reproducible sequence across sites. Restriction Spectrum Imaging (RSI) is a variant of DWI that aims to provide more information on disease characterization and increase the robustness of the acquisition. ${ }^{59}$ Compared to conventional DWI, RSI has been shown to provide improved characterization of prostate cancer in the intact setting with improved inter-reader variability. Evaluation in the metastatic setting has not yet been performed, but understanding how to evaluate heterogeneity using RSI and other novel imaging methods including ${ }^{68} \mathrm{Ga}-\mathrm{PSMA}-11$ will be important.

With emerging technologies such as MRI and next generation sequencing (NGS), we need to rethink the development and detection of aggressive prostate cancer. Though the most common solid organ malignancy in men, ${ }^{60}$ prostate cancer early detection continues to be a clinical challenge due to tumor multifocality. ${ }^{61}$ Until recently, prostate cancer diagnosis was essentially a blind procedure, where a transrectal ultrasound guided biopsy samples only about $0.04 \%$ of the prostate. ${ }^{62}$ Hence, there is no guarantee that the actual cancer focus is being sampled using ultrasound guidance. The emergence of prostate MRI, 
however, has facilitated the detection of aggressive prostate cancer with high sensitivity and negative predictive value. ${ }^{55,63,64}$ Although MRI/US fusion prostate biopsy has facilitated the longitudinal sampling of the same site of cancer, it is still unknown if low grade prostate cancer progresses to higher grade. ${ }^{65}$ It is possible that low grade cancer that "progresses" while on active surveillance may actually be a second primary. ${ }^{66}$ Additionally, prostatic intraepithelial neoplasia (PIN), which is regarded as a precursor of cancer, may actually be invasive disease. ${ }^{67}$ Additional studies are needed to delineate the existence of precancerous lesions and to determine if and how clonal progression of low to high grade cancer occurs.

\section{0 | TRANSLATING IMAGING OF HETEROGENEOUS DISEASE TO TREATMENT}

Therapeutic success in treating men with prostate cancer is limited by disease heterogeneity. In trials, patients with higher volume disease and patients with metastases perform worse when treated with targeted therapies. ${ }^{68,69}$ Clonal evolution results in a heterogeneous set of metastatic lesions within a single patient. ${ }^{11}$ Nonetheless, patientlevel genomic alterations can predict the response of patients to some therapies (eg, AR-V7). ${ }^{41,70}$ Treatment approaches that take into account disease heterogeneity across metastatic lesions are needed.

One issue limiting our ability to characterize heterogeneity, is the limited samples that are evaluated from tissue biopsies. Using PET imaging, we can label therapeutic antibodies and determine the presence of their target prior to treatment. In breast cancer this has been studied with trastuzumab, and uptake of ${ }^{89} \mathrm{Zr}$-trastuzumab prior to the initiation of therapy predicts for patient level response to therapy. ${ }^{71}$ Within the same patient, there can be a wide range of uptake across metastatic sites. ${ }^{72,73}$ Improved biomarkers are also needed to predict responses to immune checkpoint inhibitors, as the presence of PD-L1/ PD-1 can vary across lesions, ${ }^{74}$ and presence or absence of PD-L1/ PD-1 as determined by immunohistochemical staining is not always predictive of treatment response. ${ }^{75}$ Approaches using ${ }^{64} \mathrm{Cu}$-anti-PD-L1 have been performed in animal models. ${ }^{76}$ In first-in-human studies using ${ }^{89} \mathrm{Zr}$-atezolizumab (anti-PD-L1), uptake within patients varied ninefold across tumor sites, indicating a wide range of PD-L1 expression, and results on the relationship between uptake and patient level outcome are pending (NCT02453984). ${ }^{77}$ One issue with antibody-based PET imaging agents is that imaging is best performed at delayed timepoints (up to 7 days after injection), and therefore small molecule approaches would be greatly preferred. Currently there is a preclinical agent that binds to PD-L1 using ${ }^{18} \mathrm{~F}$ adnectin that has promise in allowing more feasible in vivo imaging of the heterogeneity of PD-L1. ${ }^{78}$

Once sites of metastatic disease are localized and characterized, the question remains about how to treat them. The term oligometastatic disease was coined to describe patients who have a limited number of sites of metastatic disease and therefore may be curable. ${ }^{79}$ With the increased detection of oligometastatic disease using PSMA $\mathrm{PET}$, the push to treat these lesions using metastasis-directed external beam radiation therapy has increased. ${ }^{80}$ Early phase II randomized trials have demonstrated that metastasis-directed radiation therapy can prolong ADT-free survival compared to surveillance when using choline PET/CT to detect sites of oligometastatic disease. ${ }^{81}$ Another currently ongoing trial is evaluating the benefit of stereotactic body radiation therapy (SABR) versus active clinical surveillance in patients with oligometastatic disease detected by conventional imaging (NCT02680587). ${ }^{82}$ All patients on the SABR arm receive a PSMA PET which is blinded at time of treatment, and locations of recurrence will be correlated with upfront PSMA PET to determine patterns of recurrence. These studies will help us to better understand and optimize the role of targeted radiation therapy in the setting of oligometastatic disease, and how best to use imaging to identify and manage oligometastatic disease.

\section{1 | COMPUTATIONAL MODELS TO FORECAST TUMOR PROGRESSION}

Understanding the spatiotemporal pathways of tumor progression will result in a better understanding of disease biology and has implications for patient management. For instance, Markov modeling of spatiotemporal progression pathways based on longitudinal clinical data found that in approximately $35 \%$ of metastatic breast cancer cases, the first site of metastasis is to bone, followed commonly by metastasis to the chest wall and/or lungs. ${ }^{83}$ Ultimately however, it is often liver or brain metastases which precede mortality, ${ }^{83}$ suggesting earlier support of liver health may be beneficial. Different breast cancer subtypes were also associated with particular pathways of metastatic spread and temporal progression patterns. For instance, ER-positive disease typically progresses more slowly than ER-negative disease. Combining this information with data on temporal genomic alterations may enable the development of a "clock" which describes the order and dependencies of events, and reveal progression bottlenecks and targeting opportunities. This approach is now being applied to prostate cancer.

Spatiotemporal mapping using longitudinal clinical data has also enabled classification of metastatic sites as "spreaders" or "sponges," based on the statistical probability of metastatic disease transitioning from one anatomical site to another. For instance, in breast cancer, distal lymph nodes were classified as sponges, and were not a relatively significant contributor to further metastatic spread, while in prostate cancer, lymph nodes acted as both spreaders and sponges. ${ }^{83}$ The tendency of lymph nodes in breast cancer to act more as sponges may help to explain why axillary lymph node dissection in breast cancer has been found not to improve overall survival. ${ }^{84}$ Whether or not pelvic lymph node dissection in prostate cancer impacts survival outcomes is unclear. In both prostate and breast cancer, bone metastatic sites were classified as spreaders while liver sites were classified as sponges.

Studies on metastatic tumor distribution in autopsy patients have enabled calculations of "entropy," to indicate the complexity of cancer progression. ${ }^{85}$ Compared with 12 other solid tumor types, prostate cancer had the lowest entropy score, indicating the highest 
predictability of patterns of metastatic spread. ${ }^{85}$ Approximately $80 \%$ of prostate cancers progressed through the top 30 most common twostep pathways, while only $\sim 35 \%$ of breast cancer progressed through the top 30 two-step pathways. ${ }^{85}$

Forecasting models and machine learning techniques are now being applied to predict individual patient outcomes. Compared with various statistical models using clinical data, deep learning models integrating multiple types of data including clinical and liquid biopsy data such as CTC morphometry, have been able to obtain at least 5-10\% improvement in overall performance for predictions of individual overall survival (OS) and progression free survival (PFS) (Kuhn et al, unpublished). Deep learning models also performed better than linear or Gaussian models, when using heterogeneous data (clinical, high definition single CTC imaging, and CTC enumeration), and were able to make relatively accurate individual outcome predictions (Kuhn et al, unpublished).

\section{2 | MICROENVIRONMENT: THE CANCER SWAMPS}

The tumor microenvironment (TME) is complex and can consist of a heterogeneous mix of basal cells, fibroblasts, blood vessels, immune components, nerves, extracellular matrix, etc, in addition to tumor cells. ${ }^{86}$ The makeup of the TME varies greatly from primary to metastatic cancer sites, and even within the same tissue. It has been established that there is bi-directional interplay between the tumor and $\mathrm{TME}^{87,88}$ however the molecular underpinnings are not well understood. Many histo-pathological studies have shown that cancer affects the surrounding tissue, for example bone remodeling allowing for tumor growth. ${ }^{89,90}$ Moreover, it has been shown that benign prostatic hyperplasia (BPH)-associated stroma promotes epithelial growth. ${ }^{91}$ However, less is known on a mechanistic level about how the TME promotes cancer seeding, growth and metastasis. The crosstalk between the tumor and surrounding tissue can affect cancer cell behavior and potentially therapeutic responses in patients. Some key questions include: i) how can the TME promote cancer progression; ii) how does the TME affect response to different therapies; iii) what provides a fertile soil for cancer cells to seed and metastasize; and iv) can the TME be manipulated to create a more hostile environment for cancer cells? Improved understanding of these interactions will likely lead to better predictions of therapeutic responses, as well as novel approaches targeting both the cancer and the TME to improve patient outcomes.

A first-in-field study investigated how transcriptional patterns in both tumor and stroma may promote progression of normal prostate to invasive carcinoma by isolating samples from radical prostatectomy tissue of benign, PIN and tumor regions with paired adjacent and distant stroma. ${ }^{92}$ Gene expression signatures of each region revealed that adjacent stromal signatures associated with tumor grade and outcome. In fact, stroma adjacent to areas of high Gleason score resembled a "bone homing" microenvironment, which may help to explain the preference for metastasis to the bone. Further studies are imperative to determine how altered stromal pathways can promote tumor progression.
A major hurdle in the field has been a lack of preclinical models to systematically assess tumor-TME interactions. With the development and refinement of patient-derived prostate cancer organoid models, ${ }^{93-96}$ new opportunities are arising to introduce stromal factors in these cultures and study their roles in tumorigenesis and progression. Early studies reveal that introducing stromal cells into benign prostate organoid cultures promotes organoid branching, a process similar to organogenesis (Nonn et al, unpublished). Culturing primary tumor organoids (tumoroids) with prostate stromal cells improves long-term culturing and alters stromal gene expression signatures. Questions that now can be addressed include how stroma can affect tumorigenesis and progression, for example, by culturing tumor adjacent versus distant benign stroma with normal or cancer epithelial cells, and studying morphology and other features. Moreover, epithelial and stromal cells can be specifically modified to reflect clinical alterations, which will reveal interactions between the cancer cells and TME that are dependent on their molecular characteristics.

While organoid culturing has the advantage of being able to manipulate single (ie, stromal) factors to assess their effect on tumor growth, this ex vivo approach has limitations. In vivo tissue recombination approaches could provide a more physiological context that better mimics the prostate microenvironment. ${ }^{41,97}$ Human prostatic epithelial cells can be recombined with human prostatic stromal cells and grafted under the renal capsules of mice. Manipulating both the stromal and epithelial cells will likely shed light on interplay between stromal factors and genetic alterations to promote benign prostate hyperplasia and various degrees of cancerous growth. These models often give rise to heterogeneous tumor regions within the graft, which could be used to identify TME factors that promote aggressiveness and metastasis.

In order to metastasize, cancer cells have to exit their primary site and seed a new location in the body. When regarding this process from an ecological perspective, it is possible that unfavorable conditions at the primary site (eg, hypoxia, reduced acidity) may drive cells to migrate away to find better soil, described as the optimal foraging theory, ${ }^{98}$ as opposed to disseminating tumor cells being actively attracted to other tissues. To study cancer evolution in response to changing heterogeneous environments in a controlled in vitro setting requires complex culturing conditions. With this in mind, an innovative microfluidic device was developed (Evolution Accelerator ${ }^{99}$ ), which allows gradient conditioning of the environment and can be used for single cell time-lapse microscopy. This technology enables assessment of how nutrients, therapeutics, growth factors, cancer versus stromal metabolites, and various TME components and other factors can affect cancer evolution and progression. ${ }^{100,101}$

With the new preclinical models highlighted above and others not discussed here, such as patient derived ex vivo explant modeling (PDE), ${ }^{102,103}$ new methods can be developed to study the bidirectional crosstalk between tumor and TME. Thorough clinical assessment of paired tumor and stroma in both primary and metastatic 
disease will generate new hypotheses for studying roles of stromal factors in cancer behavior and therapeutic response versus resistance.

Another aspect that should be considered is the immune component in the $\mathrm{TME}^{104}$ : what makes the prostate TME hostile to anti-tumor immune cells and how can this be overcome to make immune-based therapy (eg, PD-1 and PD-L1 targeted therapies) more effective? Some of the answers likely lie in the tumor-TME crosstalk and thus it is imperative to consider immune signaling and infiltration in these studies, especially when studying clinical samples. In sum, current efforts on studying tumor-TME communication in evolving pre-clinical models combined with clinical studies are poised to reveal novel interactions and identify new opportunities for therapeutic intervention to circumvent progression and improve patient outcomes.

\section{3 | EVALUATING IMMUNOLOGIC HETEROGENEITY}

Multiple cell types, particularly T-cells, are involved in anti-cancer immune responses. In the tumor immunity cycle, the T-cell receptor must recognize and engage the peptide-MHC complex on the tumor. Ideally, T-cells will expand, infiltrate the tumor, recognize tumor cells, and maintain functionality in the poor milieu of the tumor microenvironment which tends to diminish immune responses. This allows for antigen spreading. Effective immunotherapy needs to be able to go through this cycle-engagement, expansion, excursion, establishment of ID, and enablement, to be successful.

It is important to understand the heterogeneity of the immune response and how it changes throughout tumor progression and during treatment. Strategies for evaluating tumor and immune heterogeneity and the impact of immunotherapy in the tumor microenvironment include non-invasive methods such as ImmunoPET and peripheral blood analyses of immune cell subsets, CTCs, circulating tumor DNA, and extracellular vesicles (EVs), and invasive methods (tumor biopsies) that can assess neo-epitopes, tumor mutational burden, etc. Non-invasive techniques, such as EVs, are being developed as "liquid biopsies" to help characterize the tumor microenvironment. EVs can be isolated from blood, are released by most cell types, and can carry genetic information. ${ }^{105} \mathrm{EVs}$ are being studied as potential biomarkers and to provide information on the functional status of the tumor microenvironment. In an ongoing trial at the $\mathrm{NCl}$ evaluating the efficacy of an anti-PD-L1 antibody and a PARP inhibitor in mCRPC (NCT02484404), EV analysis of 10 patient responders versus 10 patient non-responders at day 15 found increased expression of CD45 in responders. Liquid biopsies and ImmunoPET could also provide less invasive platforms to assess heterogeneity over time, for instance to evaluate rapid adaptive responses to immune pressures. In order to understand the impact of immunotherapy on the tumor microenvironment, it is important to continue to develop tools to assay for DNA, RNA, and protein using invasive and non-invasive techniques.

\section{4 | THE ROLE OF TUMOR MUTATIONAL BURDEN IN ANTI-CANCER IMMUNE RESPONSES}

Increased tumor mutational burden is associated with increased neoantigen levels and heterogeneity and with better responses to immunotherapy. In tumor types such as non-small cell lung cancer (NSCLC), progression-free survival has been found to be significantly longer with first-line nivolumab plus ipilimumab in patients with high tumor mutational burden. ${ }^{106}$ Prostate cancer typically has a relatively low number of somatic mutations affecting protein-coding regions, and success with single agent immune checkpoint inhibition has been limited. However, mutational burden may not be a reliable biomarker for immune checkpoint inhibitors in all tumors, as some tumors with low burden of somatic mutations can be immune responsive. ${ }^{107}$ In clear cell renal cell carcinoma, tumor mutational burden was not found to be associated with response to nivolumab, but rather response was associated with distinct immune-related gene expression profiles. ${ }^{107}$ In metastatic renal carcinoma, a progression-free survival benefit during treatment with bevacizumab and atezolizumab was associated with high levels of T-effector cell function and myeloid inflammation. ${ }^{108}$ Microsatellite instability has also been associated with responsiveness to immune checkpoint inhibition, resulting in the FDA's first tissue-agnostic approval of the anti-PD-1 antibody pembrolizumab in microsatellite instability-high (MSI-H) or mismatch repair (MMR) deficient solid tumors. ${ }^{109}$ However, not all mutations are immunologic targets and immune pressure can result in enrichment for mutations that enable immune evasion. ${ }^{110}$ This has been observed in experiments with tumor cells with KRAS mutations ${ }^{111}$ and alterations in the JAK family kinases. ${ }^{110}$ Using an in vitro culture system that simulated the human tumor microenvironment, tumor cells expressing mutated KRAS were found to drive conversion of $\mathrm{CD} 4^{+} \mathrm{CD} 25^{-} \mathrm{T}$ cells into suppressive Tregs, promoting immune tolerance. ${ }^{111}$ JAK1 frameshift mutations in solid tumors were associated with high mutational burden and microsatellite instability. ${ }^{110}$ Rather than eliciting greater immune responses, JAK1 loss of function mutations were associated with tumor immune evasion through the loss of JAK1-mediated interferon responses. ${ }^{110}$ It is also important to consider that responses to immune checkpoint inhibitors in patients with high mutational burden are not universal, as was seen in MSI-H prostate cancer patients. ${ }^{112}$ Additionally, in locally advanced and metastatic urothelial carcinoma, atezolizumab showed durable activity in a phase II study in patients who had previously progressed following treatment with platinum-chemotherapy, ${ }^{113}$ but in the phase 3 IMvigor211 trial, atezolizumab was not associated with overall survival benefit versus physician's choice of chemotherapy in platinum-treated locally advanced or metastatic urothelial carcinoma with PD-L1 expression $\geq 5 \%{ }^{114}$ As evidenced by this trial, PD-L1 expression (or other checkpoints) is not a universally predictive biomarker. Similarly, neoantigen vaccine strategies need further investigation, as resident memory T-cells can vary in different tumors within a patient. ${ }^{115}$ 


\section{5 | DEFINING THE T CELLS THAT RESPOND TO CHECKPOINT IMMUNOTHERAPY}

While immune checkpoint inhibition has led to impressive responses in some tumor types, only a subset of tumor infiltrating immune cells express immune checkpoints. The clinical response to immune checkpoint blocking antibodies depends also on the nature of preexisting immunity and the tumor microenvironment. A better understanding of the types and features of immune cells within tumors will reveal strategies to improve responses to immunotherapies, and reveal how underlying immune biology contributes to disease progression and treatment responses.

$\mathrm{T}$-cell infiltration has been evaluated in a range of tumors including renal cell, prostate, and bladder. The proportion of cells in the tumor that are CD8+ T cells can vary widely within and across these different tumor types. In renal cell carcinoma, disease progression following surgery was slower in patients with higher frequencies of tumorinfiltrating CD8+ $\mathrm{T}$ cells, independent of tumor stage (Kissick et al, unpublished), supporting a role for CD8+ T cells in limiting cancer progression. Prostate tumor tissues however, had over threefold lower $\mathrm{CD} 8+\mathrm{T}$ cell numbers than renal cell carcinoma tissues, as well as lower numbers than all other cancer types evaluated (Kissick et al, unpublished).

In order to optimize the use of checkpoint immunotherapy in prostate cancer, it will be important to identify $T$ cell subsets that can be elicited with checkpoint inhibitors (most likely those that express checkpoints), and develop biomarkers that can indicate likelihood of response. In a model of chronic infection with lymphocytic choriomeningitis virus (LCMV), CD8+ T cells that proliferated in response to programmed cell death 1 (PD-1) blockade were found to be composed almost entirely of an antigen-specific PD-1-expressing subset. ${ }^{116}$ TCF7+ T cells are a subset of CD8+ cells which have a proliferative stem cell-like capacity, that normally reside in lymphoid organs in contact with antigen presenting cells (APCs) and when activated, generate effector cells which express TIM3 and other terminally differentiated markers. In human tumors, TCF7+ CD8+ T cells were found to correlate with levels of total CD8+ T cells $(H$. Kissick et al, unpublished), suggesting they maintain the $\mathrm{T}$-cell population in tumors, and might be essential for response to immune checkpoint inhibition. The levels of antigen-presenting dendritic cells (MHCll+) also correlated with the levels of TCF7+ CD8+ T cells. As the interaction between T cells and APCs is a driving factor in T cell responses, and is regulated by expression of immune checkpoints, there are significant implications for the co-localization of these populations within tumors and tumor niches in determining responses to checkpoint immunotherapy. The relatively low levels of critical anti-tumor immune populations in prostate cancer suggests a failure of immune components to effectively communicate and induce $T$ cell proliferation. Future studies must aim to identify critical immune populations, as well as detail the temporal, spatial, and numeric requirements for these cell types in driving responses to immune checkpoint blocking antibodies.

\section{6 | ROLE OF TISSUE RESIDENT MEMORY T CELLS IN TUMOR HETEROGENEITY AND RESPONSE TO CHECKPOINT IMMUNOTHERAPY}

Single-cell mass cytometry performed to characterize the tumor microenvironment in melanoma found that immune checkpoints were predominantly expressed by a small subset of CD4+ and CD8+ T-cells within the tumors, which were enriched for a tissue-resident memory $\mathrm{T}$ cell $\left(T_{R M}\right)$ phenotype. ${ }^{115} T_{R M S}$ are a distinct subset of memory $T$-cells that reside within peripheral tissues, do not recirculate, and provide immune surveillance. A marker of $T_{R M}$ is CD69, and while this is also a marker for activated T-cells, in $\mathrm{T}_{\mathrm{RM}}$ cells it is implicated in tissue retention. ${ }^{115}$ The non-circulatory, tissue-retentive properties that typically characterize $T_{R M}$ cells suggest that different $\mathrm{T}$ cell clones may inhabit different individual metastatic lesions within a patient and contribute to inter-lesional heterogeneity. To address this question, TCR sequencing was performed on multiple metastatic lesions biopsied from individual melanoma patients at the same time to compare the T-cell repertoire at different sites. ${ }^{115}$ The abundance of different T-cell clones was found to substantially vary across the different metastatic sites of individual patients, and $20 \%$ to $60 \%$ of TCRs were found to be unique to individual lesions. Data suggest TCR diversity between metastatic sites was derived from the tumor $T_{R M}$ cells. Interestingly, whole-exome sequencing of the same metastatic sites revealed that the inter-lesional diversity of TCRs was greater than the diversity of expressed somatic protein-altering single nucleotide variants (SNVs) or predicted HLA-binding epitopes. ${ }^{115}$ These data suggest $T_{R M}$ cells create genomically distinct immune microenvironments without equilibration between metastatic sites.

These findings have significant implications for monitoring responses to immunotherapy and suggest mechanisms that may contribute to observed heterogeneity of inter-lesional responses. Response and progression may be site-specific and multiple mechanisms may exist within a patient. Antigen-specificity as well as longevity of $T_{R M}$ cells may contribute to the durability of responses seen. Enhancing the frequencies of $T_{R M} s$ in tumors, targeting truncal mutations, and targeting shared and essential antigens may be potential strategies to addressing intra- and inter-lesional heterogeneity. Pluripotency genes and stemness antigens may be targets to overcome tumor heterogeneity, ${ }^{117}$ particularly in vaccine development. In addition, tumor-infiltrating lymphocyte (TIL) based therapies may benefit from harvesting $T$ cells from multiple sites of disease. The presence and role of $T_{R M}$ cells in prostate cancer remains to be determined.

\section{7 | CLINICAL TRIALS DESIGNED TO ADDRESS RESISTANCE AND HETEROGENEITY}

The past decade has seen an explosion of treatments with diverse mechanisms of action for patients with prostate cancer including targeted hormonal therapy, chemotherapy, and immunotherapy. Earlier use of agents such as docetaxel and abiraterone acetate in 
disease history is resulting in changes in disease biology and patterns of treatment resistance. As a result of the transforming standard of care, there is a need for context-focused biomarker-driven trials that enable the practice of precision medicine. Precision medicine aims to more appropriately select targeted therapies able to overcome resistance and heterogeneity, while also avoiding therapy exposure and potential toxicity in patients unlikely to derive benefit.

One challenge with delivering more precise care is consistency in defining predictive biomarkers. Two randomized phase II studies evaluating abiraterone acetate with or without PARP inhibition exemplify this challenge. ${ }^{118,119}$ In a post-hoc analysis, Hussain and colleagues found that patients with DNA repair deficient tumors had improved outcomes compared to DNA repair wild-type patients. ${ }^{118}$ In this study, DNA repair deficiency status was determined from baseline metastasis tumor biopsy using whole exome and transcriptome sequencing. ${ }^{118}$ In a separate trial, Clarke and colleagues evaluated abiraterone acetate with or without olaparib and attempted to investigate whether any added benefit of olaparib was affected by homologous recombination status. ${ }^{119}$ The biomarker subgroup analysis used to define homologous repair status was a composite analysis based on three different genomic assays, including a tissue-based assay performed on archival primary tumor tissue, a plasma-based assay for circulating tumor DNA, and a bloodbased germline assay performed on circulating leukocytes. ${ }^{119}$ Unfortunately, homologous recombination status could not be determined conclusively in $61 \%$ of patients. ${ }^{119}$ While no association was observed between homologous recombination status and treatment responses, this study was not appropriately designed and the biomarker was not clearly defined to adequately answer this question. $^{119}$

Another unmet need in the field is the definition of early stage biomarkers of response which are clinically meaningful. A recent study evaluated individual patient data, week 13 CTC response, and PSA response endpoints from five prospective randomized phase III trials that enrolled a total of 6081 patients. ${ }^{120}$ CTC nonzero at baseline and 0 at 13 weeks and CTC conversion ( $\geq 5$ CTCs at baseline, $\leq 4$ at 13 weeks) demonstrated the highest discriminatory power for overall survival and were found to represent clinically meaningful and robust response end points for early-phase metastatic CRPC clinical trials. ${ }^{120}$

For patients with localized disease, the treatment paradigm has not significantly changed over the past several decades. While radical prostatectomy is a curative treatment option for a substantial number of patients, patients with high-risk disease are at increased risk of disease recurrence and death from prostate cancer despite treatment. $^{121}$ Consequently, novel strategies utilizing multimodality therapy are warranted to improve cure rates and long term outcomes for high-risk patients. Neoadjuvant systemic therapy is utilized for the treatment of many solid tumor malignancies and has the potential to offer local and systemic disease control. ${ }^{122,123}$ Historically, neoadjuvant ADT led to improvements in the rate of organ-confined disease and decreased positive surgical margins. ${ }^{124}$ However, earlier studies failed to consistently evaluate recurrence rates and long term outcomes. More contemporary phase II neoadjuvant studies have evaluated more potent therapies targeting the AR signaling axis. Collectively, these studies have demonstrated a $10 \%$ pathologic complete response rate and $30 \%$ rate of minimum residual disease following six months of potent AR-targeting therapy. ${ }^{125-127}$ A pooled retrospective analysis of post-prostatectomy outcomes from patients enrolled on these studies demonstrated that at a median follow-up of 3.4 years, no patient with minimal residual disease experienced a disease recurrence. ${ }^{128}$

After a series of contemporary randomized phase II studies, we are at a critical cross road that warrants testing of neoadjuvant ARtargeted therapy in the context of a phase III study. However, many questions remain regarding the design of such a trial with regards to 1 ) patient selection for enrollment; 2 ) use of a biomarker to enroll patients most likely to derive benefit from intensive hormonal therapy; 3) treatment arms and inclusion of a radical prostatectomy alone arm; and 4) selection of the appropriate primary endpoint and validation of minimum residual disease as a surrogate of metastasis-free and overall survival. Additional questions remain regarding the use of the neoadjuvant paradigm to explore PARP inhibitor based therapies for patients with germline DNA repair aberrations.

For patients with non-castrate disease, the paradigm is shifting, with new objectives focused on eliminating all disease in patients previously deemed incurable with any single treatment modality. The METACURE trial (NCT03436654) which opened for accrual in June 2018 will enroll patients with high-risk localized disease, low volume metastatic disease, and biochemically recurrent disease with evidence of metastases on novel PET imaging. Patients will be treated with intense ADT and aggressive locoregional treatment including radical prostatectomy with or without lymph node dissection and/or radiation therapy. The primary endpoint will be rates of pathologic complete response and minimum residual disease.

Studies have explored mechanisms of resistance to intense androgen deprivation with leuprolide, abiraterone acetate and prednisone. Residual prostate cancer foci in radical prostatectomy specimens from patients treated with intense AR-targeted therapy were subjected to immunohistochemistry $(n=49)$ and whole exome and transcriptome sequencing $(n=18){ }^{23}$ Residual tumors demonstrated reduced but persistent nuclear AR and PSA expression. ${ }^{23}$ Additionally, Ki-67 proliferation was reduced and correlated negatively with AR activity and positively with decreased RB1 expression. RB1 genetic alterations were enriched in cases treated with intense AR-targeted therapy compared to prostate cancer cases from The Cancer Genome Atlas. ${ }^{23}$ In 15 cases where more than one tumor focus was microdissected, whole exome sequencing confirmed common clonality, however molecular alterations unique to each focus were also identified and reflect subclones found in metastatic CRPC specimens. ${ }^{23}$ Studies investigating mechanism of response and resistance to intense AR-targeted therapy with leuprolide, enzalutamide, abiraterone acetate and prednisone are currently underway. Collectively, these data shed light on mechanisms of resistance to intense neoadjuvant AR-targeted therapy. Biomarkers to identify patients most likely to benefit from this treatment strategy are needed. 


\section{8 | DISSECTING MECHANISMS OF RADIATION RESISTANCE}

Prostate cancer is generally a radiation-sensitive disease, and is responsive to treatment with external beam radiotherapy, brachytherapy, and radionuclides. However, radioresistance in prostate cancer poses a major barrier to successful treatment. Radioresistance is thought to be multifactorial, ranging from intrinsic tumor heterogeneity and cell resistance to environmental factors such as hypoxia, and both the DNA damage repair pathway and the immune environment are thought to play important roles. Targeting these two pathways are potential avenues to counter radioresistance.

The interplay between DNA damage repair (DDR) pathways and AR signaling may play an important role in the sensitization and resistance to ionizing radiation. Clinical observations suggest that the AR pathway may modulate the response to radiotherapy. Several large randomized trials have shown that addition of ADT to radiotherapy improves disease-free and overall survival in patients with prostate cancer, particularly those with high risk disease. ${ }^{129-131}$ The biologic underpinnings of this effect were not initially well understood, but several studies have begun to elucidate this mechanism. A study by Goodwin et al. demonstrated that DNA damage through ionizing radiation induces $A R$ activity, and in turn, AR induces genes required for DNA damage repair. ${ }^{132}$ Suppression of AR activity enhanced the response to DNA damage both in vitro and in vivo, suggesting a rationale for the observed clinical benefit of ADT in conjunction with radiotherapy. Using a CRPC model, Polkinghorn et al similarly showed that second-generation antiandrogen therapy downregulated DDR genes. ${ }^{133}$ Prostate cancer cell lines treated with ionizing radiation plus androgens had enhanced DNA repair, while anti-androgens caused increased DNA damage, likely through decreased classical nonhomologous end-joining, and decreased clonogenic survival. DDR gene mutations are common in prostate cancer, and these may serve as biomarkers for targeted treatment. ${ }^{41}$ However, an important point is that not all DDR genes or genetic variants are equal, and will differently impact sensitivity to radiation or targeted therapies. The functional impact of various DDR alterations in prostate cancer needs further elucidation.

The immune environment also plays a role in sensitivity and resistance to radiotherapy. Radiotherapy can enhance anti-tumor immunity through several mechanisms. Following irradiation, there is an increase in DNA damage, which leads to an increase in type I interferon (IFN) and induction of the adaptive immune response. ${ }^{134,135}$ Studies show that CD8+ T cells are required for anti-tumor effects of radiotherapy ${ }^{134}$ In murine models, anti-PD-1 antibodies can enhance antigen-specific cytokine release after radiotherapy. ${ }^{136}$ Several ongoing trials are testing the anti-tumor effects of PD-1 inhibitors combined with SBRT. A phase II trial in non-small cell lung cancer is testing whether addition of SBRT to one metastatic site prior to pembrolizumab improves outcomes compared to pembrolizumab alone (NCT02492568). Preliminary results show greater overall response rates at 12 weeks in the combination arm (41\% vs 19\%), as well as greater PFS (HR 0.55 [Cl 0.31 0.98], $P=0.04$ ) (Theelen et al, ASCO 2018, Abstract 9023).
Paradoxically, radiation can also induce immunosuppressive responses. Radiotherapy can induce PD-L1 expression through inflammatory cytokines, and can stimulate tumor pro-survival mechanisms. Radiotherapy can also generate chemotactic signals that recruit infiltration of myeloid derived suppressor cells (MDSC) and regulatory $\mathrm{T}$ cells. ${ }^{137,138}$ One promising strategy to modulate suppressive immunity and decrease radioresistance involves targeting MDSCs. The STING/IFN pathway can enhance suppressive inflammation by recruiting MDSCs in part via CCR2, a receptor for monocyte chemoattractant proteins. ${ }^{139}$ Addition of anti-CCR2 antibodies alleviates immunosuppression following activation of the STING pathway and can enhance the effects of radiotherapy in mice. ${ }^{139}$ Thus, blocking negative regulators of immunity may be a promising strategy to improve local radiotherapy.

\section{9 | THE METASTATIC PROSTATE CANCER PROJECT}

One of the challenges of studying patient tumor samples to better understand resistance and heterogeneity is that only $5 \%$ of cancer patients in the United States are enrolled in clinical trials and $85 \%$ are treated in the community setting. ${ }^{140}$ In the modern technology era, social media platforms provide a new opportunity to engage cancer patients and directly partner with them in research focused at understanding diversity within prostate cancer populations. The Metastatic Prostate Cancer Project (www.mpcproject.org) is a nationwide genomic research study for men with advanced or metastatic prostate cancer. The purpose of the platform is to generate a comprehensive database for clinical and genomic data that will be shared with the entire research community to accelerate discoveries in the field of prostate cancer research. This project was launched in collaboration with multiple advocacy partners in January 2018 and to date over 500 men have joined across the United States and Canada.

\section{0 | CONCLUSION}

The discussions at the 2018 CHPCA Meeting were productive and dynamic, with approximately 300 questions asked over 36 talks. The knowledge exchanged at this meeting through presentations, discussion, and this review article, will promote improved understandings on the mechanisms and consequences of prostate cancer heterogeneity and hopefully inspire new studies to improve prostate cancer treatment and avoid heterogeneity-driven treatment resistance and lethal disease progression.

The theme of the 2019 CHPCA Meeting will be: "Optimizing \& Accelerating Precision Medicine for Prostate Cancer."

\section{CONFLICTS OF INTEREST}

None of the other authors declare any potential conflicts of interest. 


\section{DISCLOSURE STATEMENT}

M.I.C. has served an advisory role for Pfizer. R.R.M. has served on the Advisory Board for Janssen, Novartis, and Tempus, and has received research funding from Bayer and Pfizer.

\section{ORCID}

Andrea K. Miyahira iD http://orcid.org/0000-0003-4976-002X

\section{REFERENCES}

1. Pienta KJ, Walia G, Simons JW, Soule HR. Beyond the androgen receptor: new approaches to treating metastatic prostate cancer. Report of the 2013 Prouts Neck Prostate Cancer Meeting. Prostate. 2014;74:314-320.

2. Miyahira AK, Kissick HT, Bishop JL, et al. Beyond immune checkpoint blockade: new approaches to targeting host-tumor interactions in prostate cancer: report from the 2014 Coffey-Holden Prostate Cancer Academy Meeting. Prostate. 2015;75:337-347.

3. Miyahira AK, Lang JM, Den RB, et al. Multidisciplinary intervention of early, lethal metastatic prostate cancer: Report from the 2015 CoffeyHolden Prostate Cancer Academy Meeting. Prostate. 2016;76:125-139.

4. Miyahira AK, Roychowdhury S, Goswami S, et al. Beyond seed and soil: understanding and targeting metastatic prostate cancer; Report from the 2016 Coffey-Holden Prostate Cancer Academy Meeting. Prostate. 2017;77:123-144.

5. Miyahira AK, Cheng HH, Abida W, et al. Beyond the androgen receptor II: new approaches to understanding and treating metastatic prostate cancer; Report from the 2017 Coffey-Holden Prostate Cancer Academy Meeting. Prostate. 2017;77:1478-1488.

6. Keller ET, Rowley DR, Tomlins SA, et al. Eleventh Prouts Neck Meeting on prostate cancer: emerging strategies in prostate cancer therapy. Cancer Res. 2007;67:9613-9615.

7. Ku SY, Rosario S, Wang Y, et al. Rb1 and Trp53 cooperate to suppress prostate cancer lineage plasticity, metastasis, and antiandrogen resistance. Science. 2017;355:78-83.

8. Sheahan AV, Ellis L. Epigenetic reprogramming: a key mechanism driving therapeutic resistance. Urol Oncol. 2018;36:375-379.

9. Bjurlin MA, Carter HB, Schellhammer P, et al. Optimization of initial prostate biopsy in clinical practice: sampling, labeling and specimen processing. J Urol. 2013;189:2039-2046.

10. Hong MK, Macintyre G, Wedge DC, et al. Tracking the origins and drivers of subclonal metastatic expansion in prostate cancer. Nat Commun. 2015;6:6605.

11. Gundem G, Van Loo P, Kremeyer B, et al. The evolutionary history of lethal metastatic prostate cancer. Nature. 2015;520:353-357.

12. Cooper CS, Eeles R, Wedge DC, et al. Analysis of the genetic phylogeny of multifocal prostate cancer identifies multiple independent clonal expansions in neoplastic and morphologically normal prostate tissue. Nat Genet. 2015;47:367-372.

13. Wei L, Wang J, Lampert E, et al. Intratumoral and intertumoral genomic heterogeneity of multifocal localized prostate cancer impacts molecular classifications and genomic prognosticators. Eur Urol. 2017;71:183-192.

14. VanderWeele DJ, Finney R, Katayama K, et al. Genomic heterogeneity within individual prostate cancer foci impacts predictive biomarkers of targeted therapy. Eur Urol Focus. 2018;pii:S2405S4569:30007-30015.

15. Paoletti C, Cani AK, Larios JM, et al. Comprehensive mutation and copy number profiling in archived circulating Breast cancer tumor cells documents heterogeneous resistance mechanisms. Cancer Res. 2018;78:1110-1122.

16. Augello MA, Den RB, Knudsen KE. AR function in promoting metastatic prostate cancer. Cancer Metastasis Rev. 2014;33: 399-411.

17. Huggins C. Effect of orchiectomy and irradiation on cancer of the prostate. Ann Surg. 1942;115:1192-1200.

18. Robinson D, Van Allen EM, et al. Integrative clinical genomics of advanced prostate cancer. Cell. 2015;162:454.

19. Viswanathan SR, Ha G, Hoff AM, et al. Structural alterations driving castration-resistant prostate cancer revealed by linked-read genome sequencing. Cell. 2018;174:433-447 e19.

20. Takeda DY, Spisak S, Seo JH, et al. A somatically acquired enhancer of the androgen receptor is a noncoding driver in advanced prostate cancer. Cell. 2018;174:422-432. e13.

21. Quigley DA, Dang HX, Zhao SG, et al. Genomic hallmarks and structural variation in metastatic prostate cancer. Cell. 2018;174: 758-769 e9.

22. Wu YM, Cieslik M, Lonigro RJ, et al. Inactivation of CDK12 delineates a distinct immunogenic class of advanced prostate cancer. Cell. 2018;173:1770-1782 e14

23. Sowalsky AG, $\mathrm{Ye} \mathrm{H}$, Bhasin $\mathrm{M}$, et al. Neoadjuvant-intensive androgen deprivation therapy selects for prostate tumor foci with diverse subclonal oncogenic alterations. Cancer Res. 2018;78: 4716-4730.

24. Rubio-Perez C, Tamborero D, Schroeder MP, et al. In silico prescription of anticancer drugs to cohorts of 28 tumor types reveals targeting opportunities. Cancer Cell. 2015;27:382-396.

25. Huang KL, Li S, Mertins $P$, et al. Proteogenomic integration reveals therapeutic targets in breast cancer xenografts. Nat Commun. 2017;8:14864.

26. Toska E, Osmanbeyoglu $\mathrm{HU}$, Castel $\mathrm{P}$, et al. $\mathrm{PI} 3 \mathrm{~K}$ pathway regulates ER-dependent transcription in breast cancer through the epigenetic regulator KMT2D. Science. 2017;355:1324-1330.

27. Nelson PS. Molecular states underlying androgen receptor activation: a framework for therapeutics targeting androgen signaling in prostate cancer. J Clin Oncol. 2012;30:644-646.

28. Bluemn EG, Coleman IM, Lucas JM, et al. Androgen receptor pathway-independent prostate cancer is sustained through FGF signaling. Cancer Cell. 2017;32:474-489 e6.

29. Beltran H, Prandi D, Mosquera JM, et al. Divergent clonal evolution of castration-resistant neuroendocrine prostate cancer. Nat Med. 2016;22:298-305

30. Aggarwal R, Huang J, Alumkal JJ, et al. Clinical and genomic characterization of treatment-Emergent small-Cell neuroendocrine prostate cancer: a multi-institutional prospective study. J Clin Oncol. 2018;36:2492-2503.

31. Aryee MJ, Liu W, Engelmann JC, et al. DNA methylation alterations exhibit intraindividual stability and interindividual heterogeneity in prostate cancer metastases. Sci Transl Med. 2013;5:169ra10.

32. Liu XF, Olsson P, Wolfgang CD, et al. PRAC: a novel small nuclear protein that is specifically expressed in human prostate and colon. Prostate. 2001;47:125-131.

33. Burns KH. Transposable elements in cancer. Nat Rev Cancer. 2017;17:415-424.

34. Sheaffer KL, Elliott EN, Kaestner KH. DNA hypomethylation contributes to genomic instability and intestinal cancer initiation. Cancer Prev Res (Phila). 2016;9:534-546.

35. Yegnasubramanian S, Haffner MC, Zhang Y, et al. DNA hypomethylation arises later in prostate cancer progression than $\mathrm{CpG}$ island hypermethylation and contributes to metastatic tumor heterogeneity. Cancer Res. 2008;68:8954-8967.

36. Roulois $D$, Loo Yau H, Singhania R, et al. DNA-demethylating agents target colorectal cancer cells by inducing viral mimicry by endogenous transcripts. Cell. 2015;162:961-973. 
37. Chiappinelli KB, Strissel PL, Desrichard A, et al. Inhibiting DNA methylation causes an interferon response in cancer via dsRNA including endogenous retroviruses. Cell. 2017;169:361.

38. Haffner MC, Taheri D, Luidy-Imada E, et al. Hypomethylation, endogenous retrovirus expression, and interferon signaling in testicular germ cell tumors. Proc Natl Acad Sci USA. 2018;115: E8580-E8582.

39. Weinstein IB. Addiction to oncogenes-the achilles heal of cancer. Science. 2002;297:63-64.

40. Hartwell LH, Szankasi P, Roberts CJ, Murray AW, Friend SH. Integrating genetic approaches into the discovery of anticancer drugs. Science. 1997;278:1064-1068.

41. Mateo J, Carreira S, Sandhu S, et al. DNA-repair defects and olaparib in metastatic prostate cancer. N Engl J Med. 2015;373:1697-1708.

42. Muller FL, Colla S, Aquilanti E, et al. Passenger deletions generate therapeutic vulnerabilities in cancer. Nature. 2012;488:337.

43. Dey P, Baddour J, Muller F, et al. Genomic deletion of malic enzyme 2 confers collateral lethality in pancreatic cancer. Nature. 2017; 542:119.

44. Zhao D, DePinho RA. Synthetic essentiality: targeting tumor suppressor deficiencies in cancer. BioEssays. 2017;39:1700076.

45. Zhao D, Lu X, Wang G, et al. Synthetic essentiality of chromatin remodelling factor CHD1 in PTEN-deficient cancer. Nature. 2017;542:484

46. Scher HI, Graf RP, Schreiber NA, et al. Phenotypic heterogeneity of circulating tumor cells informs clinical decisions between $A R$ signaling inhibitors and taxanes in metastatic prostate cancer. Cancer Res. 2017;77:5687-5698.

47. Scher HI, Graf RP, Schreiber NA, et al. Assessment of the validity of nuclear-Localized androgen receptor splice variant 7 in circulating tumor cells as a predictive biomarker for castration-Resistant prostate cancer. JAMA Oncol. 2018;4:1179-1186.

48. Koerber SA, Will L, Kratochwil C, et al. (68)Ga-PSMA-11 PET/CT in primary and recurrent prostate carcinoma: implications for radiotherapeutic management in 121 patients. J Nucl Med. 2018;pii: jnumed.118.211086.

49. Hamed MAG, Basha MAA, Ahmed H, Obaya AA, Afifi AHM, Abdelbary EH. (68)Ga-PSMA PET/CT in patients with rising prostatic-Specific antigen after definitive treatment of prostate cancer: detection efficacy and diagnostic accuracy. Acad Radiol. 2018;pii:S1076S6332:30262-30269.

50. van Leeuwen PJ, Donswijk M, Nandurkar R, et al. 68Ga PSMA PET/ $\mathrm{CT}$ predicts complete biochemical response from radical prostatectomy and lymph node dissection in intermediate and high-risk prostate cancer. BJU Int. 2018. https://doi.org/10.1111/bju.14506 [Epub ahead of print].

51. Seitz AK, Rauscher I, Haller B, et al. Preliminary results on response assessment using (68)Ga-HBED-CC-PSMA PET/CT in patients with metastatic prostate cancer undergoing docetaxel chemotherapy. Eur J Nucl Med Mol Imaging. 2018;45:602-612.

52. Harmon SA, Bergvall E, Mena E, et al. A prospective comparison of (18)F-Sodium fluoride PET/CT and PSMA-targeted (18)F-DCFBC PET/CT in metastatic prostate cancer. J Nucl Med. 2018;pii: jnumed.117.207373. https://doi.org/10.2967/jnumed.117.207373 [Epub ahead of print].

53. Hofman MS, Violet J, Hicks RJ, et al. [(177)Lu]-PSMA-617 radionuclide treatment in patients with metastatic castrationresistant prostate cancer (LuPSMA trial): a single-centre, singlearm, phase 2 study. Lancet Oncol. 2018;19:825-833.

54. Miyahira AK, Pienta KJ. Meeting report from the prostate cancer foundation PSMA-directed radionuclide scientific working group. Prostate. 2018;78:775-789.

55. Kasivisvanathan V, Rannikko AS, Borghi M, et al. MRI-targeted or standard biopsy for prostate-cancer diagnosis. N Engl J Med. 2018:378:1767-1777.
56. Salmasi A, Said J, Shindel AW, et al. A 17-gene genomic prostate score assay provides independent information on adverse pathology in the setting of combined multiparametric magnetic resonance imaging fusion targeted and systematic prostate biopsy. J Urol. 2018; pii:S0022-S5347:42488-42493.

57. Truong M, Feng C, Hollenberg G, et al. A comprehensive analysis of cribriform morphology on magnetic resonance imaging/ultrasound fusion biopsy correlated with radical prostatectomy specimens. J Urol. 2018;199:106-113.

58. Stoyanova R, Pollack A, Takhar M, et al. Association of multiparametric MRI quantitative imaging features with prostate cancer gene expression in MRI-targeted prostate biopsies. Oncotarget. 2016;7:53362-53376

59. White NS, McDonald C, Farid N, et al. Diffusion-weighted imaging in cancer: physical foundations and applications of restriction spectrum imaging. Cancer Res. 2014;74:4638-4652.

60. Siegel RL, Miller KD, Jemal A. Cancer statistics. CA Cancer J Clin. 2018;68:7-30.

61. Meiers I, Waters DJ, Bostwick DG. Preoperative prediction of multifocal prostate cancer and application of focal therapy: review 2007. Urology. 2007;70:3-8.

62. Fleshner NE, O'Sullivan M, Fair WR. Prevalence and predictors of a positive repeat transrectal ultrasound guided needle biopsy of the prostate. J Urol. 1997;158:505-58; discussion 508-9.

63. Siddiqui MM, Rais-Bahrami S, Turkbey B, et al. Comparison of MR/ ultrasound fusion-guided biopsy with ultrasound-guided biopsy for the diagnosis of prostate cancer. JAMA. 2015;313:390-397.

64. Ahmed HU, El-Shater Bosaily A, Brown LC, et al. Diagnostic accuracy of multi-parametric MRI and TRUS biopsy in prostate cancer (PROMIS): a paired validating confirmatory study. Lancet. 2017;389:815-822.

65. Palapattu GS, Salami SS, Cani AK, et al. Molecular profiling to determine clonality of serial magnetic resonance imaging/ultrasound fusion biopsies from men on active surveillance for low-risk prostate cancer. Clin Cancer Res. 2017;23:985-991.

66. Haffner MC, De Marzo AM, Yegnasubramanian S, Epstein JI, Carter HB. Diagnostic challenges of clonal heterogeneity in prostate cancer. J Clin Oncol. 2015;33:e38-e40.

67. Haffner MC, Weier C, Xu MM, et al. Molecular evidence that invasive adenocarcinoma can mimic prostatic intraepithelial neoplasia (PIN) and intraductal carcinoma through retrograde glandular colonization. J Pathol. 2016;238:31-41.

68. Hussain M, Tangen CM, Higano C, et al. Absolute prostate-specific antigen value after androgen deprivation is a strong independent predictor of survival in new metastatic prostate cancer: data from Southwest Oncology Group Trial 9346 (INT-0162). J Clin Oncol. 2006;24:3984-3990

69. Halabi S, Kelly WK, Ma H, et al. Meta-analysis evaluating the impact of site of metastasis on overall survival in men with castrationresistant prostate cancer. J Clin Oncol. 2016;34:1652-1659.

70. Scher HI, Lu D, Schreiber NA, et al. Association of AR-V7 on circulating tumor cells as a treatment-specific biomarker with outcomes and survival in castration-resistant prostate cancer. JAMA Oncol. 2016;2:1441-1449.

71. Gebhart G, Lamberts LE, Wimana Z, et al. Molecular imaging as a tool to investigate heterogeneity of advanced HER2-positive breast cancer and to predict patient outcome under trastuzumab emtansine (T-DM1): the ZEPHIR trial. Ann Oncol. 2016;27: 619-624.

72. Dijkers EC, Oude Munnink TH, Kosterink JG, et al. Biodistribution of 89Zr-trastuzumab and PET imaging of HER2-positive lesions in patients with metastatic breast cancer. Clin Pharmacol Ther. 2010;87:586-592.

73. Bensch F, Lamberts LE, et al. (89)Zr-Lumretuzumab PET imaging before and during HER3 antibody lumretuzumab treatment in patients with solid tumors. Clin Cancer Res. 2017;23:6128-6137. 
74. McLaughlin J, Han G, Schalper KA, et al. Quantitative assessment of the heterogeneity of PD-L1 expression in non-small-cell lung cancer. JAMA Oncol. 2016;2:46-54.

75. Broos K, Lecocq Q, Raes G, Devoogdt N, Keyaerts M, Breckpot K. Noninvasive imaging of the PD-1:PD-L1 immune checkpoint: embracing nuclear medicine for the benefit of personalized immunotherapy. Theranostics. 2018;8:3559-3570.

76. Hettich M, Braun F, Bartholoma MD, Schirmbeck R, Niedermann G. High-resolution PET imaging with therapeutic antibody-based PD-1/PD-L1 checkpoint tracers. Theranostics. 2016;6:1629-1640.

77. Bensch F, Veen Evd, Jorritsma A, et al. Abstract CT017: first-inhuman PET imaging with the PD-L1 antibody ${ }^{89} \mathrm{Zr}$-atezolizumab. Cancer Res. 2017;77:CT017-CT017.

78. Donnelly DJ, Smith RA, Morin P, et al. Synthesis and biologic evaluation of a novel (18)F-labeled adnectin as a PET radioligand for imaging PD-L1 expression. J Nucl Med. 2018;59:529-535.

79. Hellman S, Weichselbaum RR. Oligometastases. J Clin Oncol. 1995; 13:8-10.

80. Hope TA, Aggarwal R, B Chee, et al. Impact of (68)Ga-PSMA-11 PET on management in patients with biochemically recurrent prostate cancer. J Nucl Med. 2017;58:1956-1961.

81. Ost P, Reynders D, Decaestecker K, et al. Surveillance or metastasisdirected therapy for oligometastatic prostate cancer recurrence: a prospective, randomized, multicenter phase II trial. J Clin Oncol. 2018;36:446-453.

82. Radwan N, Phillips R, Ross A, et al. A phase II randomized trial of observation versus stereotactic ablative radiatlon for oligometastatic prostate cancer (ORIOLE). BMC Cancer. 2017;17:453.

83. Newton PK, Mason J, Venkatappa N, et al. Spatiotemporal progression of metastatic breast cancer: a Markov chain model highlighting the role of early metastatic sites. NPJ Breast Cancer. 2015;1:15018.

84. Giuliano AE, Ballman KV, McCall L, et al. Effect of axillary dissection vs no axillary dissection on 10-year overall survival among women with invasive breast cancer and sentinel node metastasis: the ACOSOG Z0011 (alliance) randomized clinical trial. JAMA. 2017;318:918-926.

85. Newton PK, Mason J, Hurt B, et al. Entropy, complexity, and Markov diagrams for random walk cancer models. Sci Rep. 2014;4:7558.

86. Barron DA, Rowley DR. The reactive stroma microenvironment and prostate cancer progression. Endocr Relat Cancer. 2012;19:R187-R204.

87. Cunha GR, Hayward SW, Wang YZ. Role of stroma in carcinogenesis of the prostate. Differentiation. 2002;70:473-485

88. Cunha GR, Ricke WA. A historical perspective on the role of stroma in the pathogenesis of benign prostatic hyperplasia. Differentiation. 2011;82:168-172

89. Guise T. Examining the metastatic niche: targeting the microenvironment. Semin Oncol. 2010;37:S2-14.

90. Logothetis C, Morris MJ, Den R, Coleman RE. Current perspectives on bone metastases in castrate-resistant prostate cancer. Cancer Metastasis Rev. 2018;37:189-196.

91. Barclay WW, Woodruff RD, Hall MC, Cramer SD. A system for studying epithelial-stromal interactions reveals distinct inductive abilities of stromal cells from benign prostatic hyperplasia and prostate cancer. Endocrinology. 2005;146:13-18.

92. Tyekucheva S, Bowden M, Bango C, et al. Stromal and epithelial transcriptional map of initiation progression and metastatic potential of human prostate cancer. Nat Commun. 2017;8:420.

93. Drost J, Karthaus WR, Gao D. Organoid culture systems for prostate epithelial and cancer tissue. Nat Protoc. 2016;11:347-358.

94. Beshiri ML, Tice CM, Tran C, Nguyen HM, Sowalsky AG. A PDX/ organoid biobank of advanced prostate cancers captures genomic and phenotypic heterogeneity for disease modeling and therapeutic screening. Clin Cancer Res. 2018;24:4332-4345.

95. Gao D, Vela I, Sboner A, et al. Organoid cultures derived from patients with advanced prostate cancer. Cell. 2014;159:176-187.
96. Puca L, Bareja R, Prandi D, et al. Patient derived organoids to model rare prostate cancer phenotypes. Nat Commun. 2018;9:2404.

97. Zong Y, Goldstein AS, Witte ON. Tissue recombination models for the study of epithelial cancer. Cold Spring Harb Protoc. 2015;2015: pdb top069880.

98. de Groot AE, Roy S, Brown JS, Pienta KJ, Amend SR. Revisiting seed and soil: examining the primary tumor and cancer cell foraging in metastasis. Mol Cancer Res. 2017;15:361-370.

99. Lin KC, Torga G, Wu A, et al. Epithelial and mesenchymal prostate cancer cell population dynamics on a complex drug landscape. Converg Sci Phys Oncol. 2017;3:pii: 045001. Epub 2017 Aug 30.

100. Nassar ZD, Aref AT, Miladinovic D, et al. Peri-prostatic adipose tissue: the metabolic microenvironment of prostate cancer. BJU Int. 2018;121:9-21.

101. Rycaj K, Li H, Zhou J, Chen X, Tang DG. Cellular determinants and microenvironmental regulation of prostate cancer metastasis. Semin Cancer Biol. 2017;44:83-97.

102. Centenera MM, Raj GV, Knudsen KE, Tilley WD, Butler LM. Ex vivo culture of human prostate tissue and drug development. Nat Rev Urol. 2013;10:483-487.

103. Shafi AA, Schiewer MJ, de Leeuw R, et al. Patient-derived models reveal impact of the tumor microenvironment on therapeutic response. Eur Urol Oncol. 2018;1:325-337.

104. Marciscano AE, Madan RA. Targeting the tumor microenvironment with immunotherapy for genitourinary malignancies. Curr Treat Options Oncol. 2018;19:16.

105. Morales-Kastresana A, Telford B, Musich TA, et al. Labeling extracellular vesicles for nanoscale flow cytometry. Sci Rep. 2017;7:1878.

106. Hellmann MD, Ciuleanu TE, Pluzanski A, et al. Nivolumab plus ipilimumab in lung cancer with a high tumor mutational burden. N Engl J Med. 2018;378:2093-2104.

107. Miao D, Margolis CA, Gao W, et al. Genomic correlates of response to immune checkpoint therapies in clear cell renal cell carcinoma. Science. 2018;359:801-806.

108. Atkins MB, McDermott DF, Powles T, Motzer RJ, Rini BI, Fong L. IMmotion150: a phase II trial in untreated metastatic renal cell carcinoma (mRCC) patients (pts) of atezolizumab (atezo) and bevacizumab (bev) vs and following atezo or sunitinib (sun). [ASCO abstract 4505]. J Clin Oncol. 2017;35:4505-4505.

109. FDA grants accelerated approval to pembrolizumab for first tissue/ site agnostic indication. 2017; Available from: https://www.fda.gov/ drugs/informationondrugs/approveddrugs/ucm560040.htm

110. Albacker LA, Wu J, Smith $P$, et al. Loss of function JAK1 mutations occur at high frequency in cancers with microsatellite instability and are suggestive of immune evasion. PLOS ONE. 2017;12:e0176181.

111. Zdanov S, Mandapathil M, Abu Eid R. Mutant KRAS conversion of conventional $\mathrm{T}$ cells into regulatory $\mathrm{T}$ cells. Cancer Immunol Res. 2016;4:354-365.

112. Abida W, Cheng ML, Armenia J, et al. Microsatellite instability in prostate cancer and response to immune checkpoint blockade. J Clin Oncol. 2018;36:5020.

113. Rosenberg JE, Hoffman-Censits J, Powles T, et al. Atezolizumab in patients with locally advanced and metastatic urothelial carcinoma who have progressed following treatment with platinum-based chemotherapy: a single-arm, multicentre, phase 2 trial. Lancet. 2016;387:1909-1920.

114. Powles T, Durán I, van der Heijden MS, et al. Atezolizumab versus chemotherapy in patients with platinum-treated locally advanced or metastatic urothelial carcinoma (IMvigor211): a multicentre, openlabel, phase 3 randomised controlled trial. Lancet. 2018;391:748-757.

115. Boddupalli CS, Bar N, Kadaveru K, et al. Interlesional diversity of T cell receptors in melanoma with immune checkpoints enriched in tissue-resident memory T cells. JCI Insight. 2016;1:e88955. 
116. Im SJ, Hashimoto M, Gerner MY, et al. Defining CD8+ T cells that provide the proliferative burst after PD-1 therapy. Nature. 2016;537:417.

117. Dhodapkar MV, Sexton R, Das R, et al. Prospective analysis of antigen-specific immunity, stem cell antigens and immune checkpoints in monoclonal gammopathy. Blood. 2015;126:2475-2478.

118. Hussain M, Daignault-Newton S, Twardowski PW, et al. Targeting androgen receptor and DNA repair in metastatic castration-resistant prostate cancer: results from $\mathrm{NCl}$ 9012. J Clin Oncol. 2018;36: 991-999.

119. Clarke N, Wiechno P, Alekseev B, et al. Olaparib combined with abiraterone in patients with metastatic castration-resistant prostate cancer: a randomised, double-blind, placebo-controlled, phase 2 trial. Lancet Oncol. 2018;19:975-986.

120. Heller G, McCormack R, Kheoh T, et al. Circulating tumor cell number as a response measure of prolonged survival for metastatic castration-Resistant prostate cancer: a comparison with prostateSpecific antigen across five randomized phase III clinical trials. J Clin Oncol. 2018;36:572-580.

121. Eggener SE, Scardino PT, Walsh PC. Predicting 15-year prostate cancer specific mortality after radical prostatectomy. J Urol. 2011;185:869-875.

122. Meeks JJ, Bellmunt J, Bochner BH, et al. A systematic review of neoadjuvant and adjuvant chemotherapy for muscle-invasive bladder cancer. Eur Urol. 2012;62:523-533.

123. Mauri D, Pavlidis N, loannidis JP. Neoadjuvant versus adjuvant systemic treatment in breast cancer: a meta-analysis. J Natl Cancer Inst. 2005;97:188-194.

124. McKay RR, Choueiri TK, Taplin ME. Rationale for and review of neoadjuvant therapy prior to radical prostatectomy for patients with high-risk prostate cancer. Drugs. 2013;73:1417-1430.

125. Taplin ME, Montgomery B, Logothetis CJ, et al. Intense androgendeprivation therapy with abiraterone acetate plus leuprolide acetate in patients with localized high-risk prostate cancer: results of a randomized phase II neoadjuvant study. J Clin Oncol. 2014;32:3705-3715.

126. Mostaghel EA, Nelson PS, Lange $P$, et al. Targeted androgen pathway suppression in localized prostate cancer: a pilot study. J Clin Oncol. 2014;32:229-237.

127. Montgomery B, Tretiakova MS, Joshua AM, et al. Neoadjuvant enzalutamide prior to prostatectomy. Clin Cancer Res. 2017;23: 2169-2176.

128. McKay RR, Montgomery B, Xie W, et al. Post prostatectomy outcomes of patients with high-risk prostate cancer treated with neoadjuvant androgen blockade. Prostate Cancer Prostatic Dis. 2018;21:364-372.

129. Fossa SD, Wiklund F, Klepp O, et al. Ten- and 15-yr prostate cancerspecific mortality in patients with nonmetastatic locally advanced or aggressive intermediate prostate cancer, randomized to lifelong endocrine treatment alone or combined with radiotherapy: final results of the scandinavian prostate cancer group-7. Eur Urol. 2016;70:684-691.

130. Mason MD, Parulekar WR, Sydes MR, et al. Final report of the intergroup randomized study of combined androgen-deprivation therapy plus radiotherapy versus androgen-deprivation therapy alone in locally advanced prostate cancer. J Clin Oncol. 2015;33: 2143-2150.

131. Warde P, Mason M, Ding K, et al. Combined androgen deprivation therapy and radiation therapy for locally advanced prostate cancer: a randomised, phase 3 trial. Lancet. 2011;378:2104-2111.

132. Goodwin JF, Schiewer MJ, Dean JL, et al. A hormone-DNA repair circuit governs the response to genotoxic insult. Cancer Discov. 2013;3:1254-1271.

133. Polkinghorn WR, Parker JS, Lee MX, et al. Androgen receptor signaling regulates DNA repair in prostate cancers. Cancer Discov. 2013;3:1245-1253.

134. Deng L, Liang H, Fu S, Weichselbaum RR, Fu YX. From DNA damage to nucleic acid sensing: a strategy to enhance radiation therapy. Clin Cancer Res. 2016;22:20-25.

135. Woo SR, Fuertes MB, Corrales L, et al. STING-dependent cytosolic DNA sensing mediates innate immune recognition of immunogenic tumors. Immunity. 2014;41:830-842.

136. Liang H, Deng L, Chmura S, et al. Radiation-induced equilibrium is a balance between tumor cell proliferation and T cell-mediated killing. J Immunol. 2013;190:5874-5881.

137. Vatner RE, Formenti SC. Myeloid-derived cells in tumors: effects of radiation. Semin Radiat Oncol. 2015;25:18-27.

138. Schaue D, Xie MW, Ratikan JA, McBride WH. Regulatory T cells in radiotherapeutic responses. Front Oncol. 2012;2:90.

139. Liang $H$, Deng $L$, Hou $Y$. Host STING-dependent MDSC mobilization drives extrinsic radiation resistance. Nat Commun. 2017;8:1736

140. Mills EJ, Seely D, Rachlis B, et al. Barriers to participation in clinical trials of cancer: a meta-analysis and systematic review of patientreported factors. Lancet Oncol. 2006;7:141-148.

How to cite this article: Miyahira AK, Den RB, Carlo MI, et al. Tumor cell heterogeneity and resistance; report from the 2018 Coffey-Holden Prostate Cancer Academy Meeting. The Prostate. 2019;79:244-258. https://doi.org/10.1002/pros.23729 